\title{
Cancer-induced cognitive impairment: practical solutions to reduce and manage the challenge
}

\author{
"...we recommend informing patients about the possibility of \\ cancer-induced cognitive impairment and the potential for it to worsen \\ during cancer treatment."
}

Janette L Vardy ${ }^{* 1,2,3}$, Victoria J Bray ${ }^{1,4}$ \& Haryana M Dhillon ${ }^{5}$

First draft submitted: 16 January 2017; Accepted for publication: 31 January 2017; Published online: 28 February 2017

Cancer patients coined the terms 'chemobrain' and 'chemofog' to describe cognitive symptoms they attributed to chemotherapy. More recent research has shown that cognitive impairment may be present prior to any cancer treatment $[1,2]$, and in patients who have received anticancer hormonal treatment [3]. Consequently, the term has evolved into cancer-induced cognitive impairment (CICI).

There appear to be two aspects to CICI: cognitive impairment as evaluated by formal neuropsychology tests and cognitive symptoms reported by cancer survivors. An association is consistently found between cognitive symptoms and affective symptoms, quality of life and fatigue; but these symptoms do not appear to be associated with formal neuropsychological test results in the majority of studies $[4,5]$. This is not to imply that cognitive symptoms are not real; they certainly cause some cancer survivors substantial distress and can impact many facets of their daily lives [6]. What is not known is whether patient-reported outcomes and neuropsychological tests are evaluating different constructs, or whether the weak association is due to our approach to measuring these outcomes.

The incidence of CICI in survivors of adult solid cancers is unknown. Most studies report cognitive symptoms in up to $70 \%$ of patients, with objective cognitive impairment in 30-40\% [4,7], although a small number of studies have not found any cognitive impairment post-chemotherapy. Many survivors report improvement in cognitive symptoms within $6-12$ months post treatment, although they may not return to their premorbid baseline, but for a subset of survivors the symptoms are sustained. On formal neuropsychological testing, there is a suggestion that some survivors may experience deterioration over time [8]. Crosssectional studies have shown cognitive impairment in some patients $10-20$ years after chemotherapy $[9,10]$.

The cause of CICI remains unclear but is likely multifactorial. Current hypotheses include: direct or indirect neurotoxicity from cancer treatments, including damage to progenitor cells and mitochrondria; dysregulation of the immune system; and, genetic susceptibility [11].

'Sydney Medical School, University of Sydney, Sydney, New South Wales, Australia

${ }^{2}$ Concord Cancer Centre, Concord Repatriation General Hospital, Concord, New South Wales, Australia

${ }^{3}$ Centre for Medical Psychology \& Evidence-based Decision-making, University of Sydney, Sydney, New South Wales,

Australia

${ }^{4}$ Department of Medical Oncology, Liverpool Hospital, Liverpool, New South Wales, Australia

${ }^{5}$ Centre for Medical Psychology \& Evidence-Based Decision-Making, School of Psychology, University of Sydney,

Sydney, New South Wales, Australia

*Author for correspondence: Tel.: +61 297675 000; Fax: +61 297676 342; janette.vardy@sydney.edu.au.

\section{KEYWORDS}

- cancer-induced cognitive impairment • cognition

- survivorship

“There appear to be two aspects

to cancer-induced cognitive

impairment: cognitive impairment

as evaluated by formal

neuropsychology tests and cognitive symptoms reported by cancer survivors." 


\section{"For those self-reporting ongoing difficulties with cancer-induced cognitive impairment the best current evidence supports cognitive rehabilitation programs, particularly online cognitive training."}

There is little evidence for how to prevent CICI. Early studies comparing high-dose to standard-dose chemotherapy in breast cancer patients found a dose-response, and this is supported by recent neuroimaging studies [12]. Rates of cognitive impairment with more modern regimens for breast cancer are not as high as that seen in earlier studies with the older cyclophosphamide, methotrexate, 5-flourouracil (CMF) chemotherapy regimen, but this may be due to differences in study design and analysis. There is one study suggesting a higher rate of cognitive impairment in breast cancer survivors receiving anthracylines compared with non-anthracycline regimens [13], however, there is insufficient evidence to guide chemotherapy regimen selection.

In view of the lack of robust evidence for how to treat CICI, we provide an overview of pragmatic suggestions for how to approach CICI in clinical practice.

\section{Providing patients with information about $\mathrm{CICl}$ prior to cancer treatment \& screening for $\mathrm{CICl}$ during \& after treatment}

- Cancer survivors report oncologists and other health professionals rarely mention the possibility of CICI prior to commencing cancer treatment [6,14], despite survivors telling us that knowing about this possible problem would help them prepare. Most oncologists do not screen for CICI during or after treatment. Reasons given by oncologists include: fears about priming, not believing that it is real or a common experience, and lack of knowledge about CICI and how to treat it [15];

- We recommend cognitive issues should be included in the list of side effects discussed prior to treatment and inquired about during and post treatment, in the same way fatigue is.

\section{What to do if patients report cognitive symptoms}

- In view of the strong association between cognitive and affective symptoms and fatigue, patients reporting cognitive symptoms should be assessed for anxiety, depression, fatigue, pain and sleep disturbance, and treated accordingly if present;

- Wherever possible, patients should be referred for participation in a cognitive study to better elucidate incidence and causes of CICI, and how to treat it;
- When to do neuropsychological testing? Ideally, all patients would have neuropsychological testing prior to commencing cancer treatment, with follow up as appropriate. On a practical level, the resources are not available for this in most healthcare systems, nor is it easy to schedule testing prior to starting cancer treatment. There is no simple, brief screening test with good sensitivity and specificity for CICI, and routine cognitive testing of patients is not feasible. Patients who report major decline in cognitive function during treatment, or symptoms impacting daily life that persist 6-12 months after finishing chemotherapy, or initiation of hormonal treatment, should be referred to a neuropsychologist. This enables documentation of the main areas of cognitive deficits and longitudinal assessment if appropriate;

- Potential interventions for survivors reporting cognitive symptoms: There is limited highlevel evidence for interventions to treat CICI. Here we outline potential strategies:

- Behavioral approaches:

- CICI education: Survivors with cognitive symptoms want information about CICI. Often having it validated as a real concern, and being given strategies minimizes their distress, enabling them to adapt and cope with the symptoms.

- Environmental enrichment: Environmental enrichment has been shown to be protective against CICI in a preclinical model [16]. There is lack of evidence for whether mental stimulation in cancer patients can help with CICI, but in view of the lack of negative side effects, we would encourage patients to regularly undertake mentally stimulating activities, particularly those that involve new and complex learning, such as learning a musical instrument or another language. Simpler activities such as crosswords or suduko, have a very mixed evidence base, extrapolated from non cancer populations, but do very little harm.

- Compensatory strategies: Regardless of whether the underlying cognitive deficits can be improved, patients are likely to benefit from learning to consistently 
apply simple strategies to help them adapt to any impairment. This includes simple, practical strategies such as taking notes, use of a smart phone or diary for appointments, setting alarms for reminders, minimizing distractions when working, etc. This is a similar approach to that suggested for patients with mild traumatic head injury, and can be taught by a number of health professionals including occupational therapists. However, our experience is that many people seeking advice for $\mathrm{CICI}$ are already employing compensatory strategies.

- Cognitive rehabilitation: Cognitive rehabilitation programs were initially developed for people after traumatic head injury. More recent research has investigated their use in cancer patients [17,18], although there is ongoing debate whether skills learned are transferred to 'real world' situations. Most programs focus on cognitive behavioral approaches, retraining of impaired cognitive abilities, teaching compensatory strategies, or a combination of the above. Options are to deliver the program in person, or more recently, using web-based 'brain training' programs based on adaptive exercises. Our recent randomized controlled trial evaluated a home-based, web program called Insight in 242 cancer survivors who reported persistent cognitive symptoms 6-60 months after adjuvant chemotherapy [19]. We found significant improvement in cognitive symptoms after the intervention and sustained 6 months later, compared with a standard of care control group. We also showed improvement in anxiety/depression, stress, fatigue and quality of life in the intervention arm, but no significant difference in cognitive function between the groups as measured by the computerized CogState assessment. Limited aspects of the Insight program are available on the internet free of charge or subscription to the full program can be purchased (brainhq.com). Other smaller cognitive rehabilitation studies in breast cancer survivors have found a decline in cognitive symptoms, and some have reported improvement in some aspects of objective cognitive function [20,21]. There is further work required to understand the potential translation of cognitive processing skills enhanced by these programs in real world activity and improvement in daily functioning. We recommend a trial of online cognitive rehabilitation programs for survivors with sustained cognitive symptoms after cancer treatment. They are relatively inexpensive, and web-based programs can be done from home at a time convenient to the survivor. There is some evidence to suggest that training two- to three-times per week is the optimal training time [22], but whether supervision and guidance is required is less clear. Even if cognitive training programs decrease cognitive symptoms without improvement in objective cognitive function, this outcome is important to patients.

- Exercise:

- Studies in preclinical models show exercise can prevent and improve chemotherapy-induced cognitive impairment [23]. There is preliminary evidence in cancer survivors for a benefit to $\operatorname{cog}$ nitive function from exercise, with yoga demonstrating improvement in cognitive symptoms [24]. Extrapolating from general aging and other disease groups, cognitive function has been shown to improve with exercise; aerobic and resistance-based exercise are required. Resistance training in particular, seems to increase levels of brain-derived neurotrophic factor, which is associated with better cognitive function [25];

- We recommend cancer survivors engage in regular exercise that includes aerobic, resistance and balance training; while the CICI outcomes are less clear there is no doubt that survivors will derive other benefits.

- Pharmacological agents:

- There is a lack of evidence to recommend any pharmacological intervention for CICI outside of a clinical trial. 
The neurostimulant modafinil was shown to improve some aspects of cognitive function in a small study in cancer patients with sustained fatigue [26] but further evidence in large studies is needed. A few agents such as donepezil have shown efficacy in CICI animal models [27] but have not been translated into large human studies.

In summary, we recommend informing patients about the possibility of CICI and the potential for it to worsen during cancer treatment. Treating healthcare professionals should inquire at regular intervals whether patients are experiencing symptoms of cognitive impairment. For those reporting CICI that impacts their daily living, consideration should be given to formal neuropsychological testing. Although the evidence is not currently robust for effect on
CICI, regular physical activity should be recommended as it has many other positive benefits, and there is preliminary evidence it may help CICI. Consideration could be given to participation in behavioral interventions such as education about CICI. For those self-reporting ongoing difficulties with CICI the best current evidence supports cognitive rehabilitation programs, particularly online cognitive training.

\section{Financial \& competing interests disclosure}

The authors have no relevant affiliations or financial involvement with any organization or entity with a financial interest in or financial conflict with the subject matter or materials discussed in the manuscript. This includes employment, consultancies, honoraria, stock ownership or options, expert testimony, grants or patents received or pending, or royalties.

No writing assistance was utilized in the production of this manuscript.

\section{References}

Papers of special note have been highlighted as: - of interest; $\bullet \bullet$ of considerable interest

1 Vardy J, Dhillon HM, Pond GR et al. Cognitive function and fatigue after diagnosis of colorectal cancer. Ann. Oncol. 25(12), 2404-2412 (2014).

2 Ahles TA, Saykin AJ, Mcdonald BC et al. Cognitive function in breast cancer patients prior to adjuvant treatment. Breast Cancer Res. Treat. 110(1), 143-152 (2008).

3 Schilder CM, Seynaeve C, Beex LV et al. Effects of tamoxifen and exemestane on cognitive functioning of postmenopausal patients with breast cancer: results from the neuropsychological side study of the tamoxifen and exemestane adjuvant multinational trial. J. Clin. Oncol. 28(8), 1294-1300 (2010).

- Longitudinal study showing more cognitive impairment in breast cancer survivors who did not receive chemotherapy but who received tamoxifen compared with exemestane and to noncancer controls.

Vardy JL, Dhillon HM, Pond GR et al. Cognitive function in patients with colorectal cancer who do and do not receive chemotherapy: a prospective, longitudinal, controlled study. J. Clin. Oncol. 33(34), 4085-4092 (2015)

- Large prospective longitudinal cognitive study with comprehensive testing. Patient population is colorectal cancer.

5 Hutchinson AD, Hosking JR, Kichenadasse G, Mattiske JK, Wilson C. Objective and subjective cognitive impairment following chemotherapy for cancer: a systematic review. Cancer Treat. Rev. 38(7), 926-934 (2012).

- Systematic review of cognitive impairment in cancer survivors.

6 Boykoff N, Moieni M, Subramanian SK. Confronting chemobrain: an in-depth look at survivors' reports of impact on work, social networks, and health care response. J. Cancer Surviv. 3(4), 223-232 (2009).

-• Qualitative study reporting patient experience with cognitive impairment.

7 Wefel JS, Vardy J, Ahles T, Schagen SB. International Cognition and Cancer Task Force recommendations to harmonise studies of cognitive function in patients with cancer. Lancet Oncol. 12(7), 703-708 (2011).

- Recommendations from the International Cognition and Cancer Task Force regarding methodology and establishment of a core battery.

8 Wefel JS, Saleeba AK, Buzdar AU, Meyers $\mathrm{CA}$. Acute and late onset cognitive dysfunction associated with chemotherapy in women with breast cancer. Cancer 116, 3348-3356 (2010).

9 Stouten-Kemperman MM, De Ruiter MB, Boogerd W, Veltman DJ, Reneman L, Schagen SB. Very late treatment-related alterations in brain function of breast cancer survivors. J. Int. Neuropsychol. Soc. 21(1), 50-61 (2015).
10 Koppelmans V, Breteler MM, Boogerd W, Seynaeve C, Gundy C, Schagen SB. Neuropsychological performance in survivors of breast cancer more than 20 years after adjuvant chemotherapy. J. Clin. Oncol. 30 (10), 1080-1086 (2012).

- Large study showing more cognitive impairment in breast cancer survivors 20 years after treatment compared with noncancer controls.

11 Joly F, Giffard B, Rigal O et al. Impact of cancer and its treatments on cognitive function: advances in research from the Paris International Cognition and Cancer Task Force Symposium and Update Since 2012. J. Pain Symptom Manage. 50(6), 830-841 (2015).

12 Stouten-Kemperman MM, De Ruiter MB, Koppelmans V, Boogerd W, Reneman L, Schagen SB. Neurotoxicity in breast cancer survivors $>l=10$ years post-treatment is dependent on treatment type. Brain Imaging Behav. 9(2), 275-284 (2015).

13 Kesler SR, Blayney DW. Neurotoxic effects of anthracycline- vs nonanthracycline-based chemotherapy on cognition in breast cancer survivors. JAMA Oncol. 2(2), 185-192 (2016).

14 Mitchell T, Woodward M, Hirose Y. A survey of attitudes of clinicians towards the diagnosis and treatment of mild cognitive impairment in Australia and New Zealand. Int. Psychogeriatr. 20(1), 77-85 (2008).

15 Smidt K, Mackenzie L, Dhillon H, Vardy J, Lewis J, Loh SY. The perceptions of 
Australian oncologists about cognitive changes in cancer survivors. Support. Care Cancer 24(11), 4679-4687 (2016).

Winocur G, Wojtowicz JM, Merkley CM, Tannock IF. Environmental enrichment protects against cognitive impairment following chemotherapy in an animal model. Behav. Neurosci. 130(4), 428-436 (2016). Cherrier MM, Anderson K, David D et al. A randomized trial of cognitive rehabilitation in cancer survivors. Life Sci. 93(17), 617-622 (2013).

18 Ferguson RJ, Mcdonald BC, Rocque MA et al. Development of CBT for chemotherapyrelated cognitive change: results of a waitlist control trial. Psychooncology 21(2), 176-186 (2012).

19 Bray V, Dhillon Hm, Bell M et al. Evaluation of a Web-Based cognitive rehabilitation program in cancer survivors reporting cognitive symptoms after chemotherapy. J. Clin. Oncol. 35(2), 217-225 (2016).

-• Large randomized controlled trial evaluating cognitive training.

20 Kesler S, Hadi Hosseini SM, Heckler C et al. Cognitive training for improving executive function in chemotherapy-treated breast cancer survivors. Clin. Breast Cancer 13(4), 299-306 (2013).

21 Von Ah D, Carpenter JS, Saykin A et al. Advanced cognitive training for breast cancer survivors: a randomized controlled trial. Breast Cancer Res. Treat. 135(3), 799-809 (2012).

22 Lampit A, Hallock H, Valenzuela M. Computerized cognitive training in cognitively healthy older adults: a systematic review and meta-analysis of effect modifiers. PLoS Med. 11(11), e1001756 (2014).

23 Fardell JE, Vardy J, Shah JD, Johnston IN. Cognitive impairments caused by oxaliplatin and 5-fluorouracil chemotherapy are ameliorated by physical activity. Psychopharmacology (Berl). 220(1), 183-193 (2012).

24 Vadiraja SH, Rao MR, Nagendra RH et al. Effects of yoga on symptom management in breast cancer patients: a randomized controlled trial. Int. J. Yoga 2(2), 73-79 (2009).

25 Fiuza-Luces C, Garatachea N, Berger NA, Lucia A. Exercise is the real polypill. Physiology (Bethesda) 28(5), 330-358 (2013).

26 Kohli S, Fisher SG, Tra Y et al. The effect of modafinil on cognitive function in breast cancer survivors. Cancer 115(12), 2605-2616 (2009).

27 Winocur G, Binns MA, Tannock I. Donepezil reduces cognitive impairment associated with anti-cancer drugs in a mouse model. Neuropharmacology 61(8), 1222-1228 (2011). 